\title{
Application des méthodes statistiques multivariées à l'étude hydrochimique des eaux souterraines de la région des lacs (centre de la Côte d'Ivoire)
}

\author{
Gbombélé SORO ${ }^{1 *}$, Tanina Drissa SORO ${ }^{2}$, N'guessan Marie-Rosine FOSSOU ${ }^{3}$, \\ Oi Adjiri ADJIRI ${ }^{2}$ et Nagnin SORO ${ }^{1}$ \\ ${ }^{I}$ Laboratoire des Sciences et Techniques de l'Eau et de l'Environnement, UFR Sciences de la Terre \\ et des Ressources Minières, Université Félix Houphouët-Boigny, 22 BP 582 Abidjan 22, Côte \\ d'Ivoire \\ ${ }^{2}$ Laboratoire des Sciences et Technologies de l'Environnement, UFR Environnement, Université \\ Jean Lorougnon Guédé, BP 150 Daloa Côte d'Ivoire \\ ${ }^{3}$ Laboratoire des Sciences de l'Environnement, UFR Sciences et Gestion de l'Environnement, \\ Université Nangui Abrogoua, 02 BP 801 Abidjan 02 Côte d'Ivoire \\ *Auteur correspondant ; E-mail : marc_soro@yahoo.fr, Tel : (+225) 09862685
}

\section{RESUME}

L'approvisionnement en eau potable des populations rurales est assuré par les eaux souterraines dans la région des lacs au centre de la Côte d'Ivoire. L'objectif de cette étude est de comprendre les mécanismes qui gouvernent l'hydrochimie de ces eaux. L'Analyse en Composantes Principales (ACP) et la Classification Ascendante Hiérarchique (CAH) ont été appliquées aux données chimiques de 94 forages d'eau échantillonnés. La température moyenne des eaux est de $27,21^{\circ} \mathrm{C}$ avec un $\mathrm{pH}$ moyen de 6,6 . La conductivité électrique moyenne est de $331 \mu \mathrm{S} / \mathrm{cm}$ avec des valeurs extrêmes comprises entre 11,2 et $1097 \mu \mathrm{S} / \mathrm{cm}$. Ces eaux sont aptes à la consommation en dehors de certains points d'eau dont les concentrations en nitrates, en fer et en manganèse sont supérieures aux normes OMS. Trois composantes principales cumulent une variance totale de 75\% des 13 variables. L'ACP et la CAH mettent en évidence trois processus hydrogéochimiques dans l'évolution hydrochimique des eaux souterraines. L'hydrolyse acide des silicates et des carbonates secondaires produit les ions $\mathrm{Ca}^{2+}, \mathrm{Mg}^{2+}, \mathrm{SO}_{4}{ }^{2-}$ et $\mathrm{HCO}_{3}{ }^{-}$dans les eaux. L'oxydo-réduction semble être à l'origine du fer et du manganèse. Les concentrations de $\mathrm{NO}_{3}{ }^{-}$et de $\mathrm{Cl}^{-}$sont liées aux activités anthropiques et à la décomposition de la matière organique. L'hydrochimie des eaux souterraines est régit par les facteurs naturels et anthropogéniques.

(C) 2019 International Formulae Group. All rights reserved

Mots clés: Analyse en Composantes Principales (ACP), Classification Ascendante Hiérarchique (CAH), processus, hydrochimie, eaux souterraines, Côte d'Ivoire.

\section{Application of multivariate statistical methods for hydrochemical assessment of groundwater in lakes region (centre of Côte d'Ivoire)}

\begin{abstract}
Groundwater resources supply the rural inhabitants in drinking water within Lakes region of Côte d'Ivoire. The main objective was to apply Principal Component Analysis (PCA) and Cluster analysis (CA) to
\end{abstract}


assess the main processes that are responsible for the hydrochemistry of groundwater. Ninety-four (94) boreholes were sampled for quality assessment. Overall, the water samples are suitable for drinking. Nevertheless, some boreholes have iron, manganese and nitrates higher than the World Health Organization (WHO) permissible limits. Three principal components explain $75 \%$ of the total variance of the 13 parameters. The PCA and CA revealed that the hydrochemistry evolution of groundwater was mainly controlled by the weathering and hydrolysis of silicates, redox and anthropogenic activities. The hydrolysis acid of silicates produces the major part of the ions in groundwater such as $\mathrm{Ca}^{2+}, \mathrm{Mg}^{2+}, \mathrm{SO}_{4}{ }^{2-}$ and $\mathrm{HCO}_{3}^{-}$. Besides, redox was another factor that produces $\mathrm{Mn}$ and $\mathrm{Fe}$ in groundwater. In addition, anthropogenic activities produce $\mathrm{NO}_{3}{ }^{-}$and $\mathrm{Cl}^{-}$in groundwater. In fact, both natural and anthropogenic factors characterized the hydrochemistry evolution within the study area.

(C) 2019 International Formulae Group. All rights reserved

Keywords: Principal Component Analysis (PCA), Cluster Analysis (CA), processes, hydrochemistry, groundwater, Côte d'Ivoire.

\section{INTRODUCTION}

Dans la plupart des régions du monde, l'eau souterraine est d'une importance capitale. Elle l'est encore plus dans les zones rurales d'Afrique pour diverses raisons. L'eau souterraine est généralement moins chère et les aquifères jouissent d'une protection naturelle contre les pollutions anthropiques (Calow et al., 2010). Elle est également une source d'approvisionnement fiable et un tampon contre la sécheresse (Calow et al., 2010). En Côte d'Ivoire, et particulièrement dans la région des Lacs, les eaux souterraines sont quasiment destinées à l'alimentation en eau potable des populations rurales nonobstant la présence de nombreuses retenues d'eau et barrages qui sont réservés à l'agriculture et à l'hydroélectricité. En effet, elle est douce et de bonne qualité comparée à celle de surface. Malgré cette importance, la qualité de l'eau souterraine peut se détériorer du fait de nombreux facteurs influençant sa composition chimique et par conséquent ses multiples usages. Parmi ces facteurs, on peut citer entre autres la lithologie, le temps de résidence de l'eau au contact des roches, la température ambiante et le $\mathrm{pH}$, la composition chimique de l'aquifère, les conditions climatiques qui prévalent durant la formation, la quantité de l'eau disponible dans l'aquifère et son taux de circulation (Akoteyon, 2013). En plus de ces processus naturels, les activités anthropiques peuvent aussi influencer les caractéristiques hydrogéochimiques des eaux souterraines
(Yuan et al., 2017). La combinaison de tous ces facteurs dans un système complexe crée plusieurs types d'eau qui évoluent aussi bien dans l'espace que dans le temps. L'hydrochimie constitue donc un excellent outil d'investigation de la structure et du fonctionnement des aquifères. Dans ce cas, les paramètres physico-chimiques et chimiques sont alors utilisés comme des traceurs naturels. L'utilisation des traceurs naturels chimiques constitue à la fois un moyen d'investigation indirect précieux et une alternative pour mieux appréhender la dynamique des systèmes aquifères complexes (Alaya et al., 2014).

Les processus influençant

l'hydrochimie des eaux souterraines étant très variés, leurs études requièrent donc nécessairement l'application des méthodes multivariées. Les techniques statistiques multivariées ont été utilisées dans la littérature pour faciliter la résolution des problèmes géoenvironnementaux. Ces techniques ont été utilisées avec succès pour permettre de comprendre les systèmes d'écoulement des eaux souterraines (Cloutier et al., 2008; Yidana et al., 2011) et les processus influençant l'hydrochimie des eaux souterraines à l'échelle du bassin et à l'échelle régionale (Cloutier et al., 2008, Yidana et al., 2010 ; Soro et al., 2013; Amadou et al., 2014 ; Yuan et al., 2017). Les méthodes statistiques multivariées ont été aussi utilisées pour identifier et mettre en lumière les sources 
majeures d'effets anthropogéniques sur la qualité des eaux souterraines et de surface (Jiang et al., 2009 ; Kim et al., 2009 ; Yidana et al., 2011). La compréhension des propriétés hydrauliques des couches aquifères et la caractérisation hydrochimique de l'eau qu'elles contiennent sont nécessaires pour la planification et la gestion des ressources en eau souterraine. En effet, le rejet de certains points d'eau par les populations est souvent dû aux propriétés physico-chimiques de l'eau. Ainsi un excès de minéralisation naturelle peut donner à l'eau un gout salé. De même, une coloration rougeâtre de l'eau est observée dans certains cas où le fer est en excès. A cette minéralisation naturelle intrinsèque à la nature des roches traversées par les forages, il convient d'y adjoindre la minéralisation acquise liée à l'impact des activités anthropiques telle que l'agriculture. La qualité des eaux souterraines est donc sous l'influence de nombreux facteurs géologiques et anthropogéniques. L'objectif de cette étude est d'utiliser les techniques statistiques multivariées telles que l'Analyse en Composantes Principales (ACP) et la Classification Hiérarchique Ascendante (CHA) afin de comprendre les processus hydrogéochimiques qui gouvernent les eaux souterraines dans la région des Lacs (Centre de la Côte d'Ivoire).

C'est dans cette optique que s'inscrit la présente étude. Elle se base sur l'utilisation des techniques d'analyses statistiques multivariées à savoir l'Analyse en Composantes Principales (ACP) et la Classification Ascendante Hiérarchique (CAH) pour comprendre les processus qui gouvernent les eaux souterraines de la région des Lacs (Centre de la Côte d'Ivoire).

\section{MATERIEL ET METHODES}

\section{Présentation de la zone d'étude}

La zone d'étude se situe dans la région des Lacs, Centre de la Côte d'Ivoire, entre les latitudes $6^{\circ} 30$ et $7^{\circ} 35$ Nord et les longitudes $4^{\circ} 40$ et $5^{\circ} 40$ Ouest (Figure 1). Au plan administratif, la région des Lacs a pour principale ville, Yamoussoukro, capitale politique du pays. Elle couvre une superficie d'environ $6000 \mathrm{~km}^{2}$ pour une population estimée en 2014 à 502489 habitants (Institut National de la Statistique, 2014). La zone est drainée par le fleuve Bandama et les affluents du N'zi et est dotée de nombreuses retenues d'eau destinées à l'agriculture. C'est une large pénéplaine entourée par les monts du Yaouré au Nord-Ouest et la chaîne de Fétékro au SudEst, qui culminent respectivement à $623 \mathrm{~m}$ à l'Ouest du barrage de Kossou et à $646 \mathrm{~m}$ au Sud-Est de Yamoussoukro (Leblond, 1984). L'altitude moyenne de la région comprise entre ces deux massifs est de $200 \mathrm{~m}$, la plaçant ainsi à la limite méridionale de la zone dite des plateaux (200 et $500 \mathrm{~m}$ ). Les altitudes les plus élevées sont voisines de $240 \mathrm{~m}$. Le climat est de type équatorial de transition atténué (climat baouléen), entre les climats de type guinéen et de type soudanien (Leblond, 1984). Il est caractérisé par quatre saisons dont deux saisons sèches et deux saisons pluvieuses. Du point de vue géologique (Figure 1), la région des Lacs appartient au domaine protérozoïque. Elle est située dans le birimien de la Côte d'Ivoire qui appartient au domaine «BaouléMossi» du craton ouest africain. Les principales formations géologiques sont constituées de roches magmatiques et métamorphiques (Leblond, 1984 ; N'guessan, 1985 ; Yacé, 2002). Les roches magmatiques appartiennent au complexe éburnéen et comprennent les granitoïdes éburnéens. Ce sont des granites à biotite, des granites à deux micas, des migmatites, des granodiorites concordants et des pegmatites. Les formations volcano-sédimentaires sont représentées par les schistes (vert et ardoisier), les quartzites, les métasédiments indifférenciés et les roches vertes du birimien. Les roches vertes sont des roches basiques dont la nature originelle est encore décelable et des roches complètement transformées correspondant aux faciès amphibolite et schiste vert (Soule de Lafont, 
1956). Ce sont des schistoïdes caractérisées par une légère schistosité et de structure granoblastique. Elles se fissurent mais ne se délitent pas et se comportent comme des schistes (Engalenc, 1978). La couleur verte à l'affleurement leur a conféré ce nom générique de roches vertes. Ainsi sous ce vocable, on y trouve des métabasaltes, des métaandésites, des métadolérites et des métagabbros (Yacé, 2002). Sur le terrain, elles se présentent sous forme allongées, étroites avec un relief défavorable à l'implantation de sites de villages (N'guessan, 1985). Ces deux principales formations géologiques conduisent à la formation d'un contexte hydrogéologique caractérisé par deux types d'aquifères : un aquifère d'altérites et un aquifère fissuré ou fracturé. L'aquifère de fissure ou de fracture est exploité par des forages dont la profondeur atteint rarement les $100 \mathrm{~m}$ (Soro et al., 2010). C'est l'aquifère le plus sollicité dans les projets d'alimentation en eau potable des populations rurales en Côte d'Ivoire. Mais la productivité des ouvrages hydrauliques varie de manière aléatoire. Compte tenu de leurs perméabilité et porosité relativement faibles, la productivité de ces aquifères est modeste. Dans cette région, les forages ont un débit moyen de $2,32 \mathrm{~m}^{3} / \mathrm{s}$ avec des valeurs de transmissivité variant de $1,15 \cdot 10^{-6}$ à $4,48 \cdot 10^{-4}$ $\mathrm{m}^{2} / \mathrm{s}$ (Soro et al., 2010). Au niveau hydrostructurale, la direction N130 à N150 est dominante dans les ensembles granitiques. Par contre, la direction N020 à N030 est toujours bien représentée et est caractéristique des formations volcano-sédimentaires du birimien. Dans ce bassin, les couches sont orientées selon une direction N40-50, dite direction Baoulé. Tous ces accidents jouent un rôle très important dans la mise en place des aquifères de fractures ou de fissures (Soro et al., 2010). Ils peuvent constituer également des couloirs de contamination des nappes par les polluants.

\section{Données}

Les données de cette étude ont été fournies par la Direction Territoriale de l'Hydraulique (DTH) de Yamoussoukro. Ce sont des analyses faites sur les eaux de nouveaux forages réalisés dans la zone d'étude dans le cadre du projet Caisse Française de Développement et le Conseil de l'Entente phase 3 (CFD/CE3) en 1998 et 2000. Les analyses chimiques des eaux ont été faites à l'aide d'un DR $3000 \mathrm{HACH}$. Les paramètres physico-chimiques concernés sont: la température de l'eau $\left(\mathrm{T}^{\circ} \mathrm{C}\right)$, la conductivité électrique (CE) et le pH. Les éléments majeurs analysés sont les bicarbonates $\left(\mathrm{HCO}_{3}^{-}\right)$, le titre alcalimétrique complet (TAC), le titre hydrométrique total (THT), le calcium $\left(\mathrm{Ca}^{2+}\right)$, le magnésium $\left(\mathrm{Mg}^{2+}\right)$, les chlorures $\left(\mathrm{Cl}^{-}\right)$, le fer $(\mathrm{Fe})$, le manganèse $(\mathrm{Mn})$, les nitrates $\left(\mathrm{NO}_{3}^{-}\right)$, les sulfates $\left(\mathrm{SO}_{4}^{2-}\right)$. Un conductimètre et un pHmètre de marque Hanna Instruments (HI 9033 Multi-range) ont servi à mesurer in situ le $\mathrm{pH}$, la conductivité électrique (CE) et la température $\left(\mathrm{T}^{\circ} \mathrm{C}\right)$. Au total 94 points d'eaux ont été échantillonnés (Figure 2).

\section{Méthodes}

L'approche méthodologique est basée sur l'utilisation des méthodes statistiques multivariées que sont l'analyse en composantes principales normées (ACPN) et la classification ascendante hiérarchique $(\mathrm{CAH})$. Les méthodes statistiques multivariées ont été utilisées pour identifier et mettre en lumière les sources majeures des effets anthropogéniques sur la qualité des eaux souterraines et de surface (Jiang et al., 2009 ; Kim et al., 2009; Yidana et al., 2011).

\section{Analyse en Composantes Principales (ACP)}

L'analyse en composantes principales (ACP) est une méthode statistique 
multidimensionnelle permettant de synthétiser les informations dans le but de comparer les systèmes entre eux. L'ACP est particulièrement utilisée et adaptée (Hussein, 2004 ; Ahoussi et al., 2008 ; Sojka et al., 2008 ; Soro, 2010 ; Monjerezi et al., 2012 ; Yuan et al., 2017) pour expliquer d'une part les ressemblances chimiques entre les différentes eaux et/ou les différents pôles d'acquisition de la minéralisation et d'autre part les variables qui gouvernent ces mécanismes. En effet, c'est une technique qui permet de prendre en compte un grand nombre de variables et d'échantillons. Par ailleurs, l'ACP est une technique qui présente une forte sensibilité aux valeurs extrêmes (Garry, 2007). Cette forte sensibilité aux extrêmes impose d'appliquer l'ACP sur des données centrées réduites ou normalisées qui donnent le même poids à chaque variable. Pour ce faire les données d'entrée ont été normalisées (Equation 1). Cette étape est très importante dans l'ACP car au niveau du calcul des distances euclidiennes, les variables avec de fortes variances tendent à avoir une grande influence sur celles qui ont des variances faibles (Güler et al., 2002; Cloutier et al., 2008).

$$
z=\frac{x-\mu}{\sigma}
$$

Où $\mathrm{z}$ est la valeur normalisée ; $\mathrm{x}$ est la donnée. $\mu$ et $\sigma$. sont respectivement la moyenne et l'écart-type des données. L'analyse a porté sur un total de 94 échantillons avec 13 paramètres $\left(\mathrm{T}^{\circ} \mathrm{C}\right.$, Conductivité, $\mathrm{pH}, \mathrm{TAC}, \mathrm{THT}, \mathrm{Ca}^{2+}$, $\mathrm{Mg}^{2+}, \mathrm{HCO}_{3}^{-}, \mathrm{NO}_{3}^{-}, \mathrm{Cl}^{-}, \mathrm{SO}_{4}{ }^{2-}, \mathrm{Mn}$ et $\mathrm{Fe}$ ).

\section{Classification Ascendante Hiérarchique (CAH)}

L'analyse de classification (Cluster Analysis) comprend un ensemble de techniques statistiques qui sont utilisées pour déterminer des groupes statistiques naturels ou des structures dans les données. Selon Hussein
(2004), l'application de cette méthode en géologie est récente. Cette méthode est donc couramment utilisée pour analyser les données hydrochimiques des eaux et vient en appoint de l'ACP (Hussein, 2004). C'est un outil puissant pour l'analyse des données chimiques des eaux compte tenu de la complexité des systèmes hydrochimiques et des difficultés que l'on rencontre quant à leur interprétation. Le fondement mathématique de la méthode tel que proposé par Hussein (2004) est basé sur le calcul de la distance euclidienne entre les individus ou observations dans un espace à $n$ dimensions. Premièrement, les données doivent être normalisées par le calcul de leurs moyennes à l'aide l'équation 2 suivante.

$K_{i j}=\frac{\left(X_{i j}-X\right)}{S_{i c}}$

où $\mathrm{K}_{\mathrm{ij}}$ est la valeur normale de $\mathrm{X}_{\mathrm{ij}}$ pour la $\mathrm{i}^{\text {ième }}$ variable du $\mathrm{j}^{\text {ième }}$ individu, $\mathrm{X}$ est la moyenne de la i ième variable et $S_{\text {ic }}$ l'écart type.

La procédure adoptée donne un poids égal à chaque variable. Ainsi, la mesure de similarité est tout simplement la distance définie dans un espace euclidien (Hussein, 2004).

La distance entre deux individus $(\mathrm{j}, \mathrm{k})$ est donnée par l'équation 3 suivante :

$$
\mathrm{d}_{\mathrm{ij}}=\left[\sum_{i=1}^{N}\left(\mathrm{~K}_{\mathrm{ij}}-\mathrm{K}_{\mathrm{ik}}\right)^{2}\right]^{1 / 2}
$$

où $\mathrm{K}_{\mathrm{ik}}$ représente la $\mathrm{K}_{\mathrm{ième}}$ variable mesurée sur l'objet $\mathrm{i}$, et $\mathrm{K}_{\mathrm{jk}}$ la $\mathrm{K}^{\text {ième }}$ variable mesurée sur l'objet j.

Le résultat est donné sous forme d'un dendrogramme horizontal ou vertical qui classe les observations ou variables par groupes ou sous-groupes ayant le même poids ou les mêmes caractéristiques. Cette méthode permet donc de faire un regroupement des observations ou variables en fonction des similarités qui existent entre celles-ci ou non. L'analyse statistique des données obtenues a été faite avec le logiciel Statistica 6.0 StatSoft France (2003). 


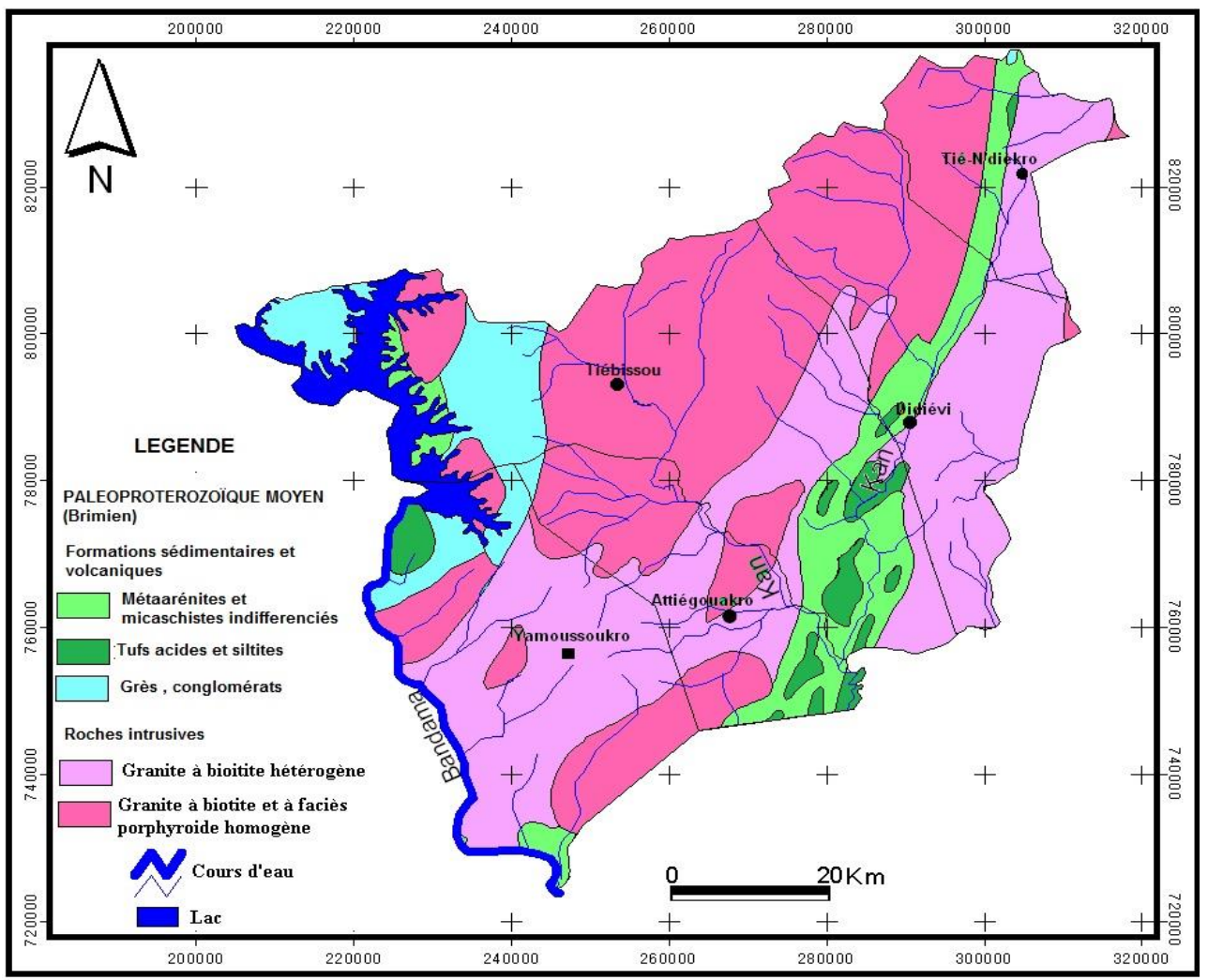

Figure 1 : Carte de localisation et géologique de la zone d'étude.

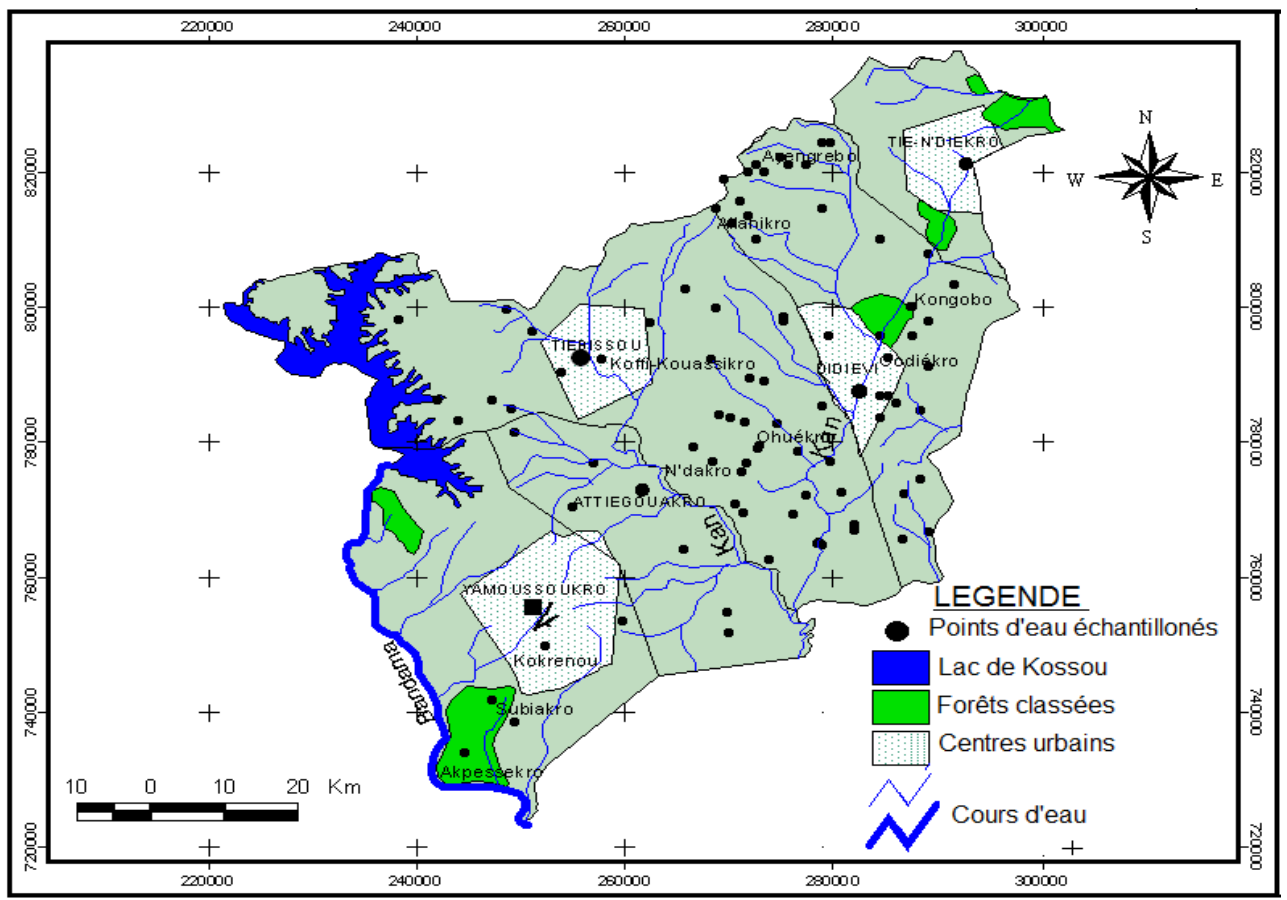

Figure 2 : Carte des points d'échantillonnage des forages de la région des lacs. 


\section{RESULTATS}

L'analyse de la balance ionique entre les cations et les anions a été calculée. Elle est en moyenne moins de $5 \%$ pour l'ensemble des données démontrant ainsi la fiabilité des résultats d'analyse.

\section{Caractérisation hydrochimique des eaux souterraines de la région des lacs}

L'analyse statistique des paramètres physico-chimiques des échantillons d'eaux souterraines de la région des lacs est résumée dans le Tableau 1. Les températures des eaux souterraines de la région des lacs sont supérieures à la norme de potabilité dans les forages. Toutes ces valeurs sont audessus de la valeur guide de l'OMS $\left(25^{\circ} \mathrm{C}\right)$ comme valeur impérative pour tenir compte des circonstances climatiques dans les eaux destinées à la consommation humaine. La température enregistrée les eaux varie de 25,50 à $30,10{ }^{\circ} \mathrm{C}$ avec un écart type de 0,91 ${ }^{\circ} \mathrm{C}$ et une moyenne de $27,21^{\circ} \mathrm{C}$. Les valeurs les plus fréquentes sont situées entre 26 et $27{ }^{\circ} \mathrm{C}$ et entre 27 et $28{ }^{\circ} \mathrm{C}$. Ces deux intervalles représentent plus de $65 \%$.

Les eaux sont pour la plupart légèrement acides à alcalines avec des valeurs de $\mathrm{pH}$ qui varient de 5,58 à 8,04 avec une moyenne de $6,6 \pm 0,577$. Les valeurs les plus fréquentes se situent dans l'intervalle de la norme de potabilité soit plus de $70 \%$ des ouvrages. Cependant, $30 \%$ des ouvrages ont des valeurs comprises entre 4 et 6 . Les eaux de ces ouvrages sont acides et ne sont pas aptes à la consommation humaine.

Les conductivités mesurées varient de 11,2 à $1097 \mu \mathrm{S} / \mathrm{cm}$ avec une moyenne de $331,17 \pm 172,45 \mu \mathrm{S} / \mathrm{cm}$. Les valeurs de conductivité les plus fréquentes sont comprises entre 10 et $200 \mu \mathrm{S} / \mathrm{cm}$ et 200 et 400 $\mu \mathrm{S} / \mathrm{cm}$ soit $65 \%$ des valeurs. Ces valeurs se situent dans la norme recommandée pour les eaux d'alimentation en eau potable par l'OMS qui est de $400 \mu \mathrm{S} / \mathrm{cm}$. Cependant, 35\% des ouvrages ont des conductivités supérieures à cette valeur guide. Les eaux de ces ouvrages ont un goût salé.
L'azote est présent dans les eaux sous sa forme nitrate $\left(\mathrm{NO}_{3}^{-}\right)$. Les valeurs varient de 0 à $149,40 \mathrm{mg} / \mathrm{L}$ avec une moyenne de 3,56 \pm $1,49 \mathrm{mg} / \mathrm{L}$. Les valeurs les plus fréquentes sont comprises entre 0 et $10 \mathrm{mg} / \mathrm{L}$. Les ouvrages de Subiakro et d'Akpessekro présentent respectivement les concentrations les plus élevées $149,40 \mathrm{mg} / \mathrm{L}$ et $105,6 \mathrm{mg} / \mathrm{L}$. Ces valeurs sont supérieures aux normes établies fixées à $50 \mathrm{mg} / \mathrm{L}$. Les valeurs de sulfate $\mathrm{SO}_{4}{ }^{2-}$ varient de 0 à $30 \mathrm{mg} / \mathrm{L}$ avec une moyenne de 4,99 $\pm 5,68 \mathrm{mg} / \mathrm{L}$. Les valeurs les plus fréquentes dans la région sont celle comprises entre 0 et $5 \mathrm{mg} / \mathrm{L}$. Les chlorures varient de $1 \mathrm{mg} / \mathrm{L}$ à $117 \mathrm{mg} / \mathrm{L}$, avec une moyenne de $15,64 \pm 1,41 \mathrm{mg} / \mathrm{L}$. Les teneurs les plus fréquentes sont comprises entre 0 et 20 $\mathrm{mg} / \mathrm{L}$ soit environ $80 \%$ des échantillons. Les plus fortes valeurs enregistrées vont de 45 $\mathrm{mg} / \mathrm{L}$ à $117 \mathrm{mg} / \mathrm{L}$. Les bicarbonates varient de $35 \mathrm{mg} / \mathrm{L}$ à $325 \mathrm{mg} / \mathrm{L}$. Les concentrations sont très variables avec une moyenne de 139,92 $\mathrm{mg} / \mathrm{L}$. Les concentrations les plus fréquentes sont situées entre 50 et $100 \mathrm{mg} / \mathrm{L}$ et entre 150 et $200 \mathrm{mg} / \mathrm{L}$. Les anions se présentent selon l'ordre d'abondance suivant : $\mathrm{HCO}_{3}{ }^{-}>\mathrm{Cl}^{-}$ $>\mathrm{SO}_{4}{ }^{2-}>\mathrm{NO}_{3}{ }^{-}$.

Les concentrations en $\mathrm{Ca}^{2+}$ varient de 4,01 mg/L dans le forage d'Attekro 2 à 105,8 1 $\mathrm{mg} / \mathrm{L}$ dans celui de N'Dié. La très forte valeur de l'écart type $(20,82 \mathrm{mg} / \mathrm{L})$ traduit une grande variation des concentrations avec une moyenne de 30,24 mg/L. La quasi-totalité des points d'eau de la région des lacs présentent des concentrations inférieures à la norme de potabilité des eaux d'alimentation à l'exception d'un seul forage dont la concentration est légèrement supérieure à la norme. Les concentrations les plus fréquentes se situent entre 0 et $20 \mathrm{mg} / \mathrm{L}$ et entre 20 et 40 $\mathrm{mg} / \mathrm{L}$ et représentent environ $70 \%$ des échantillons. Aucune valeur de $\mathrm{Mg}^{2+}$ ne dépasse la norme de potabilité définie pour les eaux d'alimentation. Par conséquent, ces eaux ne présentent pas de risques potentiels à l'homme. Les concentrations varient de 0,49 $\mathrm{mg} / \mathrm{L}$ à 35,96 mg/L. Ces données ne sont pas très dispersées car l'écart type observé $(6,98$ $\mathrm{mg} / \mathrm{L}$ ) est faible avec une moyenne de 8,49 $\mathrm{mg} / \mathrm{L}$. Les concentrations les plus fréquentes 
dans les eaux se situent entre 0 et $5 \mathrm{mg} / \mathrm{L}$ et 5 et $10 \mathrm{mg} / \mathrm{L}$ soit environ $70 \%$ des échantillons d'eaux.

Les valeurs minimales du fer et du manganèse sont nulles. Les valeurs moyennes sont supérieures à la norme de potabilité définie par l'OMS. $84 \%$ des échantillons ont des concentrations comprises entre 0 et 0,03 $\mathrm{mg} / \mathrm{L}$, ces valeurs sont inférieures à la norme de potabilité définie contre, $16 \%$ qui ont des concentrations dépassant la norme de potabilité. Au niveau du manganèse, $80 \%$ des échantillons ont des concentrations comprises entre 0 et $0,05 \mathrm{mg} / \mathrm{L}$. $20 \%$ des échantillons présentent des concentrations qui excèdent la valeur de $0,05 \mathrm{mg} / \mathrm{L}$. Les concentrations les plus élevées en fer : 1,$72 ; 1,73 ; 3,96 ; 6,31$ et $16,19 \mathrm{mg} / \mathrm{L}$. Au niveau du manganèse, les concentrations au-dessus de la valeur guide vont de $0,25 \mathrm{mg} / \mathrm{L} ; 0,6 \mathrm{mg} / \mathrm{L} ; 1,4 \mathrm{mg} / \mathrm{L}$ et de $2,7 \mathrm{mg} / \mathrm{L}$. La présence de ces deux éléments en concentrations élevées est liée au caractère réducteur des eaux qui favorise leur libération. Ces deux ions sont souvent à la base de la coloration rougeâtre de l'eau de certains forages.

Les valeurs du Titre Hydrométrique Total et du Titre Alcalimétrique Complet sont comprises entre 2 et $38^{\circ} \mathrm{F}$ et entre 0,7 et 6,5 ${ }^{\circ} \mathrm{F}$ respectivement. Leurs valeurs moyennes sont de $10,95{ }^{\circ} \mathrm{F}$ et de $2,79{ }^{\circ} \mathrm{F}$ respectivement. Dans l'ensemble, les résultats indiquent que les eaux souterraines de la région sont des eaux douces et moyennement minéralisées.

\section{Analyse en Composantes Principales de l'hydrochimie des eaux souterraines}

La corrélation entre deux éléments est très bonne si elle est proche de 1 . Le Tableau 2 donne les différentes corrélations qui existent entre les éléments chimiques analysés dans les eaux. A l'analyse de ce tableau, on constate qu'il existe une très bonne corrélation entre le $\mathrm{HCO}_{3}^{-}$et le TAC $(0,993)$. Cette corrélation très significative indique que l'essentiel de l'alcalinité des eaux provient des bicarbonates. Le Titre Hydrotimétrique Totale présente une très bonne corrélation avec le calcium $(0,959)$ et le magnésium $(0,847)$. La dureté totale des eaux est gouvernée ici par l'apport en calcium et en magnésium. Le fer et le manganèse présentent une bonne liaison entre eux $(0,890)$ parce qu'ils sont sous le contrôle d'un même processus chimique qui permet leur mise en solution l'oxydoréduction. La conductivité électrique (CE) est corrélée respectivement avec le THT $(0,885)$, le calcium $(0,892)$, à un degré moindre avec le $\mathrm{HCO}_{3}^{-}(0,767)$, le TAC $(0,777)$ et le $\mathrm{SO}_{4}{ }^{2-}(0,732)$. La conductivité est également moyennement corrélée avec le magnésium $(0,664)$ et avec les chlorures $(0,664)$. Ces différentes corrélations indiquent que la minéralisation des eaux provient non seulement de l'hydrolyse des silicates. Le calcium est corrélé avec le TAC $(0,786)$ et le $\mathrm{HCO}_{3}^{-}(0,780)$. On note également une corrélation entre le THT et $\mathrm{HCO}_{3}^{-}(0,753)$ et TAC $(0,759)$. Ces corrélations traduisent l'abondance de ces ions par rapport aux autres dans la minéralisation des eaux. Elles indiquent également que le processus chimique majoritaire qui gouverne ces eaux est la dissolution des formations silicatées. La très faible corrélation qui existe entre les chlorures et les nitrates indique que les nitrates ont une origine superficielle (pollution anthropique).

Le Tableau 3 représente les cinq premiers facteurs avec leurs valeurs propres et les différents pourcentages exprimés. On constate que les 5 premiers facteurs expriment à eux seuls $89,14 \%$ de l'information dont $46,72 \%$ pour le facteur $1,15,30 \%$ pour le facteur 2 et $13,44 \%$ pour le facteur 3 . Les trois premiers facteurs totalisent $75,46 \%$ de la variance totale exprimée. Le couple F1-F2 exprime à lui seul plus de $60 \%$ de l'information. Au vue de ces pourcentages exprimés, les mécanismes qui contrôlent l'évolution chimique des eaux de la région sont largement contenus dans ces trois facteurs. De ce fait, l'analyse portera uniquement sur ces trois facteurs dans la mesure où l'ACP d'une région n'est valable que si le pourcentage de variance totale cumulée est supérieur à $70 \%$.

Les vecteurs propres permettant de définir chacun de ces trois facteurs par rapport aux variables sont reportés dans le Tableau 4. 
Ce tableau a permis de construire des cercles de corrélation entre les trois premiers facteurs. Le cercle de corrélation du plan factoriel F1F2 (Figure 3) montre que le facteur F1 est déterminé dans sa partie négative par le groupe de variables $\mathrm{Ca}^{2+}, \mathrm{Mg}^{2+}$, THT, TAC, conductivité électrique (CE), $\mathrm{HCO}_{3}{ }^{-}$et $\mathrm{SO}_{4}{ }^{-}$. Les variables telles que $\mathrm{HCO}_{3}{ }^{-}, \mathrm{Ca}^{2+}$,

$\mathrm{Mg}^{2+}$, sont en général issues de l'altération des roches et de l'hydrolyse acide des minéraux silicatés. Ce phénomène étant un processus lent lié à la nature des formations géologiques, au degré de fracturation et d'altération de la roche. Ceci est valable dans le cadre de cette étude. En effet, les forages échantillonnés sont réalisés dans les aquifères de fissures où les vitesses d'écoulement des eaux sont faibles dû à un réseau de fractures peu développé en région de socle. Dans ce cas, l'eau reste dans le massif plus longtemps et peut donc atteindre un degré d'équilibre chimique élevé avec la roche encaissante. De ce fait, le facteur F1 rend compte des conditions d'acquisition de la minéralisation. Il exprime donc le temps de séjour des eaux dans l'aquifère et le mécanisme d'acquisition de la minéralisation des eaux. Ces processus se déroulent naturellement dans l'aquifère. C'est pourquoi, ce facteur est appelé minéralisation naturelle par hydrolyse acide des silicates-temps de séjour. Le facteur 2 est surtout représentatif du fer $\left(\mathrm{Fe}^{2+}\right.$ et du manganèse $\left(\mathrm{Mn}^{2+}\right)$. La proximité entre ces deux éléments sur l'axe 2 signifie qu'ils sont mis en solution par le même mécanisme chimique qui est ici l'oxydo-réduction. L'origine de ces deux éléments dans les eaux est liée aux conditions aérobies. La présence du fer et du manganèse serait liée au milieu réducteur que constitue l'aquifère captif. Le caractère réducteur des eaux favoriserait donc la libération de ces deux éléments. La position de ces éléments dans la partie négative du facteur 2 est aussi interprétée comme un processus géochimique et biogéochimique naturel des eaux. Le facteur F2 exprime donc un phénomène d'oxydo-réduction qui favoriserait la mise en solution du fer et du manganèse.
Dans le plan factoriel F1-F3 (Figure 4), le facteur F3 est caractérisé par l'opposition des variables $\mathrm{Cl}^{-}$et $\mathrm{NO}_{3}^{-}$dans la partie positive avec le $\mathrm{pH}$. Lorsque le $\mathrm{pH}$ diminue, donc lorsque le milieu devient acide, les eaux sont chargées en $\mathrm{Cl}^{-}$et $\mathrm{NO}_{3}^{-}$. La proximité apparente de $\mathrm{Cl}^{-}$et $\mathrm{NO}_{3}^{-}$témoigne d'une origine commune de ces deux ions et ne doit pas faire illusion sur le faible coefficient de corrélation qui les lie $(r=0,506)$. Bien qu'il soit faible, il demeure le meilleur qu'on puisse trouver entre ces deux ions puisque l'essai dans d'autres plans factoriels ne permet pas de mieux les analyser. La présence de ces deux éléments dans les eaux souterraines est attribuée pour ce qui est des chlorures au pluviolessivage de la voûte forestière et des sols humifères et aux activités anthropiques (utilisation des engrais) pour les nitrates $\left(\mathrm{NO}_{3}{ }^{-}\right)$. Le facteur $\mathrm{F} 3$ exprime l'origine spatiale des éléments par infiltration directe des eaux superficielles et l'influence des activités anthropiques sur la qualité de l'eau.

\section{Classification Ascendante Hiérarchique de l'hydrochimie des eaux souterraines}

Les résultats de la classification ascendante hiérarchique sont présentés par le dendrogramme de la Figure 5. A l'analyse de graphe, on distingue deux grandes classes statistiques distinctes. La première est déterminée par $\mathrm{HCO}_{3}^{-}$et la CE. Dans cette classe, on remarque l'influence prépondérante de $\mathrm{HCO}_{3}^{-}$sur la minéralisation des eaux souterraines. La deuxième classe statistique peut être subdivisée en deux sous-classes à partir de cette analyse statistique :

- une classe qui comprend la Température et le $\mathrm{Ca}^{2+}$. Elle représente l'effet de la température sur le processus de précipitation de la calcite et de l'altération des silicates par hydrolyse ;

- une classe composée de trois groupes qui sont en relation avec le phénomène d'altération par hydrolyse des silicates et celui de l'infiltration superficielle d'éléments d'origine anthropique :

- un groupe composé des éléments $\mathrm{SO}_{4}{ }^{2-}$ et $\mathrm{pH}$ lié au phénomène de dissolution de la matière organique et des minéraux tels que 
l'arsénopyrite dont la dissolution produit des sulfates ;

- un groupe qui comprend $\mathrm{NO}_{3}{ }^{-}, \mathrm{Mn}$ et Fe. Les nitrates sont liés à une pollution d'origine anthropique. Le fer et le manganèse sont issus du phénomène d'oxydo-réduction ;

- un groupe avec les éléments TAC, THT, $\mathrm{Mg}^{2+}$ et dans une moindre mesure $\mathrm{Cl}^{-}$. Les trois premiers éléments sont liés au phénomène d'hydrolyse des silicates. Les chlorures sont issus d'une infiltration des précipitations.

\section{Origine des ions bicarbonates, calcium et magnésium dans les eaux}

L'analyse de l'hydrochimie des eaux souterraines a montré que le $\mathrm{Ca}^{2+}, \mathrm{Mg}^{2+}$ et $\mathrm{HCO}_{3}{ }^{-}$sont les ions dominant dans les eaux souterraines. De même, en se référant à la matrice de corrélation (Tableau 2), on note que le bicarbonate est bien corrélé avec la conductivité $(\mathrm{r}=0,767)$. Cette affinité traduit l'influence qu'à ce dernier sur la minéralisation totale dans les eaux souterraines. De ce fait, le bicarbonate se positionne comme le meilleur indicateur des concentrations dissoutes en solution. La Figure 6 permet d'analyser l'influence des bicarbonates sur la minéralisation des eaux. On remarque qu'une grande partie du nuage de point est déporté vers l'axe des bicarbonates, indiquant la prépondérance des bicarbonates sur la charge saline des eaux. Cependant, certains points tendent vers l'axe de la conductivité traduisant une participation non négligeable des autres ions à la salinisation. Par ailleurs, le faible coefficient de corrélation linéaire $\left(\mathrm{R}^{2}=0,62\right)$ indique que le bicarbonate n'est pas le seul ion qui conditionne la minéralisation des eaux bien qu'étant dominant. La Figure 7 indique une corrélation linéaire faible $\left(\mathrm{R}^{2}=0,65\right)$ entre $\mathrm{Ca}^{2+}$ et $\left(\mathrm{HCO}_{3}{ }^{-}+\mathrm{SO}_{4}{ }^{2-}\right)$. De plus, la majorité des points est déporté vers le pôle $\mathrm{HCO}_{3}{ }^{-}+$ $\mathrm{SO}_{4}{ }^{2-}$ indiquant qu'une grande partie des concentrations en calcium observées dans les eaux est issue de l'hydrolyse des carbonates secondaires (aragonite, calcite et dolomite). Le graphe de la Figure 8 montre qu'il existe une corrélation linéaire moyenne $\left(\mathrm{R}^{2}=0,64\right)$ entre $\left(\mathrm{Ca}^{2+}+\mathrm{Mg}^{2+}\right)$ et $\left(\mathrm{SO}_{4}{ }^{2-}+\mathrm{HCO}_{3}^{-}\right)$. Le coefficient de détermination ainsi que l'ordonnée à l'origine de la droite de régression semble indiquer qu'une partie de calcium et de magnésium pourrait bien provenir de la dissolution des carbonates secondaires tels que l'aragonite, la calcite et la dolomite de la matrice aquifère.

Tableau 1: Analyse statistique des paramètres physico-chimiques des eaux souterraines.

\begin{tabular}{lccccc}
\hline Paramètres & Minimum & Maximum & Moyenne & Ecart-type & OMS (1994) \\
\hline $\mathbf{T}$ & 25,5 & 30,1 & 27,21 & 0,91 & $<25$ \\
$\mathbf{p H}$ & 5,58 & 8,04 & 6,6 & 0,577 & $6,5<\mathrm{pH}<9,5$ \\
$\mathbf{C E}$ & 11,2 & 1097 & 331,17 & 172,45 & $<400$ \\
$\mathbf{C a}^{2+}$ & 4,01 & 105,81 & 30,24 & 20,82 & 100 \\
$\mathbf{M g}^{2+}$ & 0,48 & 35,96 & 8,49 & 6,98 & 50 \\
$\mathbf{H C O}^{-}$ & 35 & 325 & 139,92 & 71,33 & \\
$\mathbf{N O}_{3}{ }^{-}$ & 0 & 149,4 & 3,56 & 16,49 & 50 \\
$\mathbf{M n}$ & 0 & 2,7 & 0,07 & 0,28 & 0,05 \\
$\mathbf{F e}$ & 0 & 16,19 & 0,38 & 1,62 & 0,3 \\
$\mathbf{T H T}$ & 2 & 38 & 10,95 & 7,28 & \\
$\mathbf{T A C}$ & 0,7 & 6,5 & 2,79 & 1,42 & \\
$\mathbf{S O} \mathbf{~}^{2-}$ & 0 & 30 & 4,99 & 5,68 & 250 \\
$\mathbf{C l}^{-}$ & 1 & 117,02 & 15,64 & 15,81 & 200 \\
\hline
\end{tabular}

Les paramètres physico-chimique sont en $\mathrm{mg} / \mathrm{L}$ à l'exception du $\mathrm{pH}$, de la $\mathrm{T}$ en ${ }^{\circ} \mathrm{C}$, de Conductivité Electrique (CE) en : $\mu \mathrm{S} / \mathrm{cm}$, le Titre hydrométrique Total (THT) et le titre alcalimétrique complet (TAC) en $\left({ }^{\circ} \mathrm{F}\right)$ 
Tableau 2 : Matrice de corrélation entre les éléments physico-chimiques des eaux souterraines de la région des Lacs.

\begin{tabular}{llllllllllllll}
\hline & $\boldsymbol{T}^{\circ} \boldsymbol{C}$ & $\boldsymbol{p H}$ & $\boldsymbol{C E}$ & $\boldsymbol{H C O 3}$ & $\boldsymbol{T A C}$ & $\boldsymbol{T H T}$ & $\boldsymbol{C a}$ & $\boldsymbol{M g}$ & $\boldsymbol{C l}$ & $\boldsymbol{F e}$ & $\boldsymbol{M n}$ & $\boldsymbol{N O 3}$ & $\boldsymbol{S O 4}$ \\
\hline$T^{\circ} C$ & 1,000 & & & & & & & & & & & & \\
$p H$ & $-0,257$ & 1,000 & & & & & & & & & & & \\
$C E$ & $-0,011$ & 0,414 & 1,000 & & & & & & & & & & \\
$H C O 3$ & $-0,153$ & 0,678 & $\mathbf{0 , 7 6 7}$ & 1,000 & & & & & & & & & \\
$T A C$ & $-0,134$ & 0,674 & $\mathbf{0 , 7 7 7}$ & $\mathbf{0 , 9 9 3}$ & 1,000 & & & & & & & & \\
$T H T$ & $-0,073$ & 0,371 & $\mathbf{0 , 8 8 5}$ & $\mathbf{0 , 7 5 3}$ & $\mathbf{0 , 7 5 9}$ & 1,000 & & & & & & & \\
$C a$ & $-0,088$ & 0,428 & $\mathbf{0 , 8 9 2}$ & $\mathbf{0 , 7 8 0}$ & $\mathbf{0 , 7 8 6}$ & $\mathbf{0 , 9 5 9}$ & 1,000 & & & & & & \\
$M g$ & $-0,032$ & 0,179 & 0,664 & 0,529 & 0,533 & $\mathbf{0 , 8 4 7}$ & 0,661 & 1,000 & & & & & \\
$C l$ & 0,069 & $-0,047$ & 0,664 & 0,188 & 0,194 & 0,519 & 0,496 & 0,438 & 1,000 & & & & \\
$F e$ & 0,221 & $-0,132$ & $-0,120$ & $-0,092$ & $-0,088$ & $-0,112$ & $-0,109$ & $-0,093$ & $-0,105$ & 1,000 & & & \\
$M n$ & 0,203 & $-0,107$ & $-0,097$ & $-0,061$ & $-0,063$ & $-0,099$ & $-0,084$ & $-0,102$ & $-0,113$ & $\mathbf{0 , 8 9 0}$ & 1,000 & & \\
NO3 & 0,142 & 0,025 & 0,367 & 0,074 & 0,078 & 0,163 & 0,190 & 0,076 & 0,506 & $-0,094$ & $-0,073$ & 1,000 & \\
$S O 4$ & 0,030 & 0,397 & $\mathbf{0 , 7 3 2}$ & 0,666 & 0,669 & 0,637 & 0,678 & 0,412 & 0,509 & 0,030 & 0,059 & 0,223 & 1,000 \\
\hline
\end{tabular}

Tableau 3 : Valeurs propres et pourcentage de variance exprimée.

\begin{tabular}{llllll}
\hline & $\mathrm{F} 1$ & $\mathrm{~F} 2$ & $\mathrm{~F} 3$ & $\mathrm{~F} 4$ & $\mathrm{~F} 5$ \\
\hline Valeurs propres & 6,07 & 1,99 & 1,75 & 0,97 & 0,81 \\
\% Variance exprimée & 46,72 & 15,30 & 13,44 & 7,47 & 6,21 \\
\% Variance exprimée cumulée & 46,72 & 62,02 & 75,46 & 82,93 & 89,14 \\
\hline
\end{tabular}

Tableau 4: Vecteurs propres (coefficients de corrélation des variables centrées réduites avec les facteurs).

\begin{tabular}{|c|c|c|c|c|c|}
\hline \multirow[b]{2}{*}{ Variable } & \multicolumn{5}{|c|}{ Facteur } \\
\hline & F1 & F2 & F3 & F4 & F5 \\
\hline $\mathrm{T}^{\circ} \mathrm{C}$ & 0,1076 & $-0,4512$ & 0,3750 & $-0,0625$ & 0,7957 \\
\hline $\mathrm{pH}$ & $-0,5555$ & 0,1772 & $-0,5467$ & $-0,4264$ & 0,0371 \\
\hline Cond & $-0,9464$ & $-0,0762$ & 0,1846 & $-0,0277$ & $-0,0194$ \\
\hline $\mathrm{HCO}_{3}$ & $-0,8830$ & 0,0052 & $-0,3692$ & $-0,1153$ & 0,1087 \\
\hline TAC & $-0,8872$ & $-0,0016$ & $-0,3583$ & $-0,1139$ & 0,1242 \\
\hline THT & $-0,9364$ & $-0,0492$ & 0,0630 & 0,2878 & $-0,0043$ \\
\hline $\mathrm{Ca}$ & $-0,9327$ & $-0,0503$ & 0,0150 & 0,1243 & $-0,0129$ \\
\hline $\mathrm{Mg}$ & $-0,7271$ & $-0,0349$ & 0,1356 & 0,5271 & 0,0099 \\
\hline $\mathrm{Cl}$ & $-0,5442$ & $-0,0951$ & 0,6988 & $-0,0054$ & $-0,2464$ \\
\hline $\mathrm{Fe}$ & 0,1630 & $-0,9177$ & $-0,2084$ & 0,0135 & $-0,1688$ \\
\hline $\mathrm{Mn}$ & 0,1395 & $-0,9162$ & $-0,2254$ & $-0,0245$ & $-0,1811$ \\
\hline $\mathrm{NO}_{3}$ & $-0,2642$ & $-0,0522$ & 0,6342 & $-0,5853$ & $-0,1497$ \\
\hline $\mathrm{SO}_{4}$ & $-0,7772$ & $-0,2207$ & 0,0368 & $-0,1968$ & 0,0089 \\
\hline
\end{tabular}




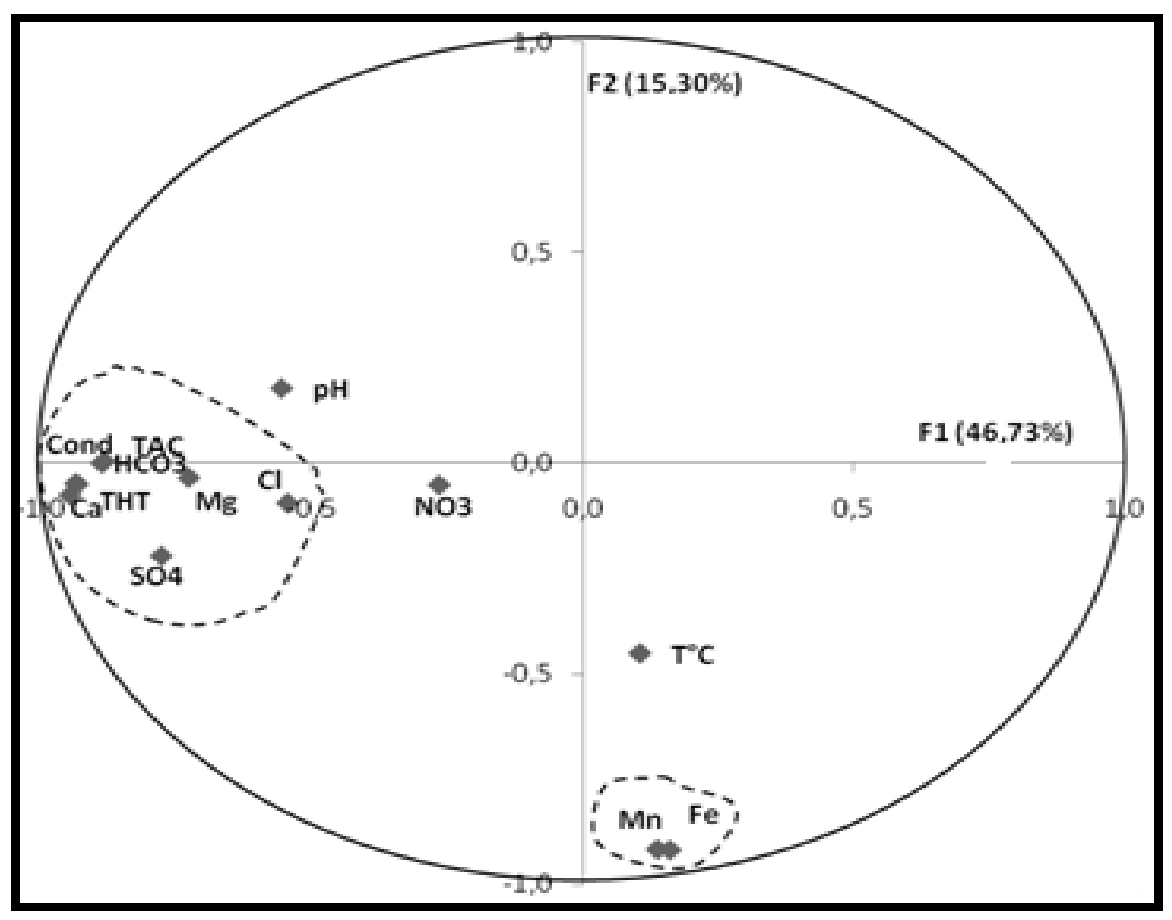

Figure 3: Composantes principales des variables chimiques des eaux souterraines dans le plan F1F2.

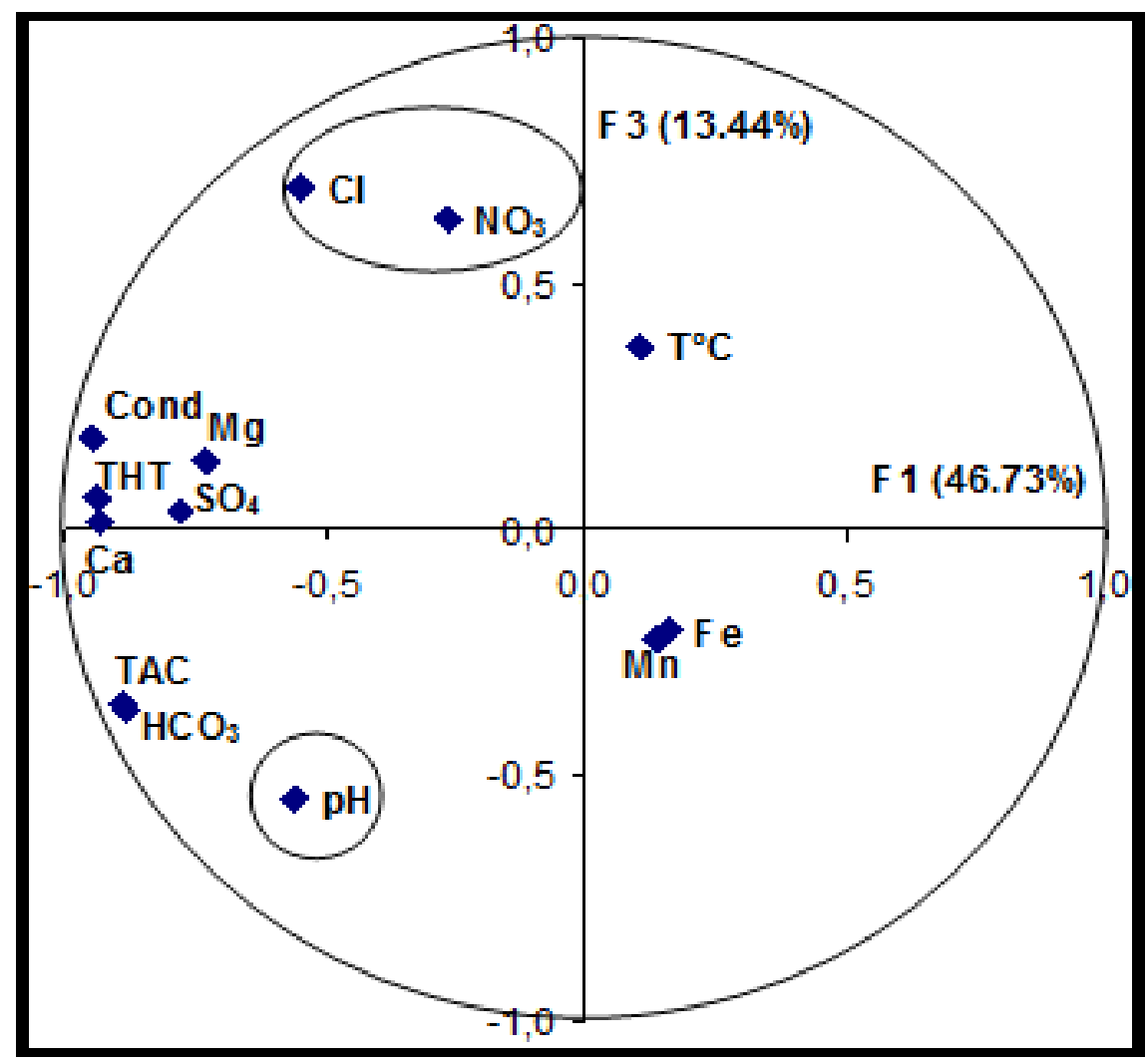

Figure 4: Composantes principales des variables chimiques des eaux souterraines dans le plan F1F3. 


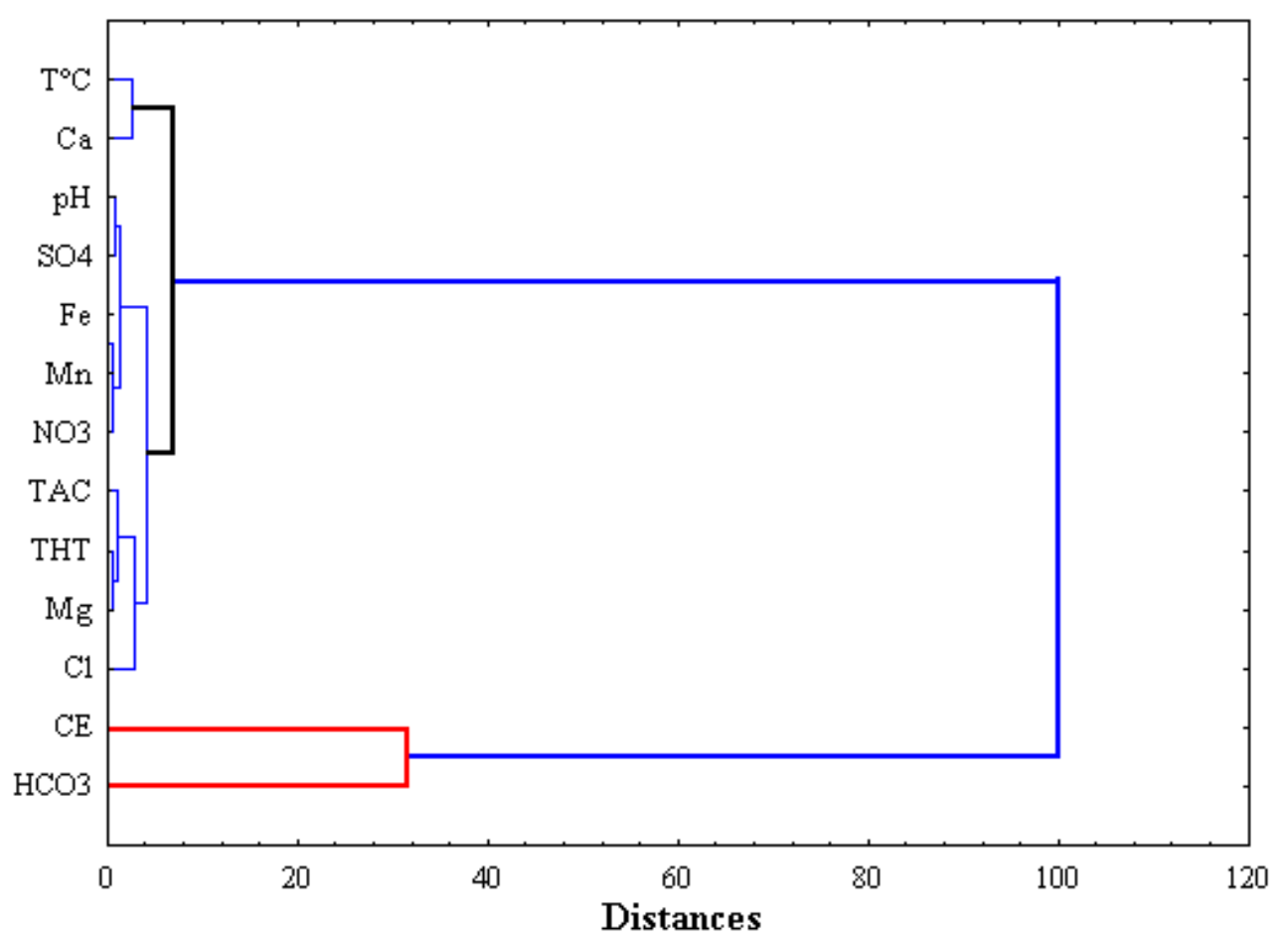

Figure 5: Dendrogramme des variables chimiques des eaux souterraines.

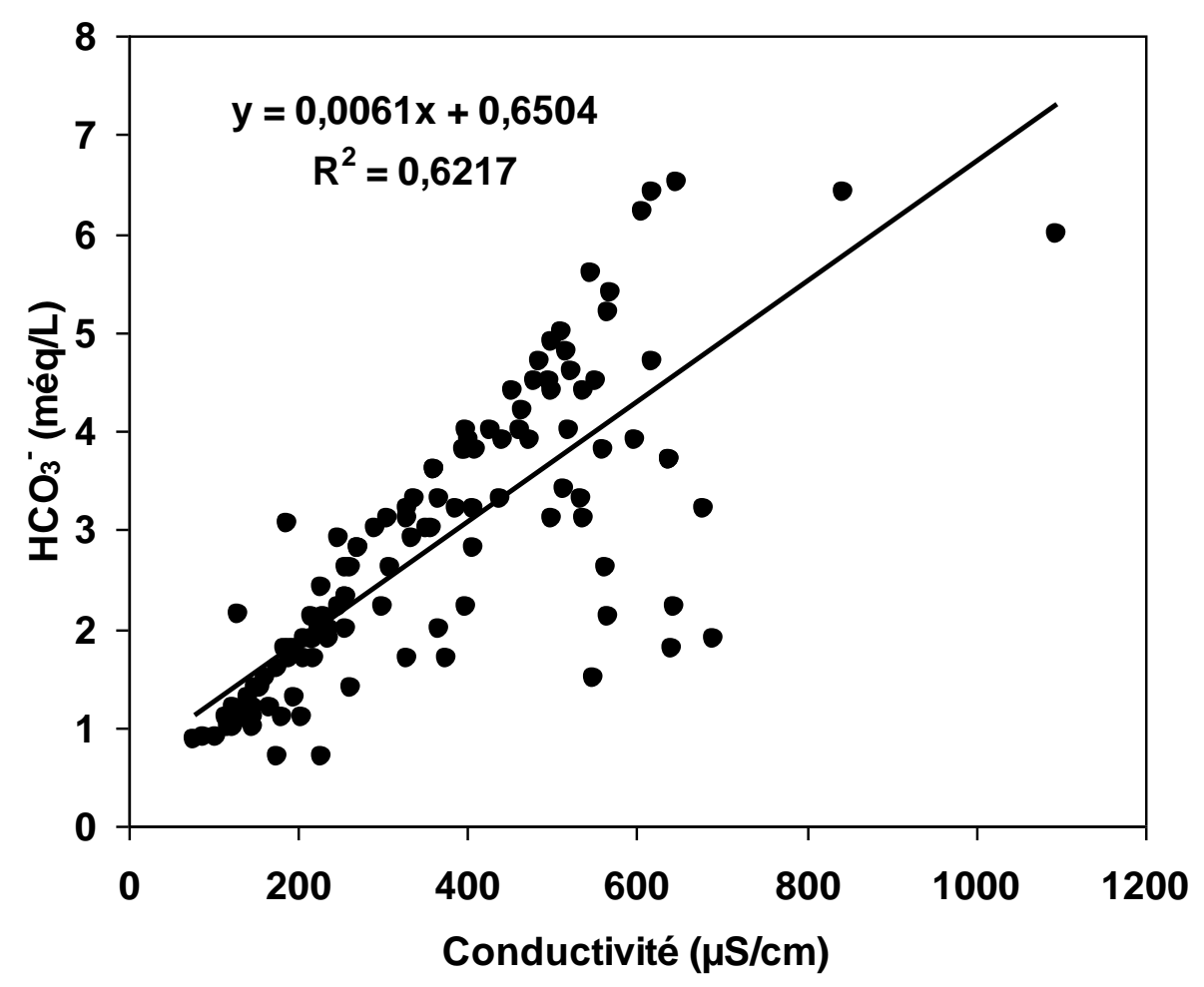

Figure 6: Graphe de corrélation entre la conductivité et $\mathrm{HCO}_{3}$. 


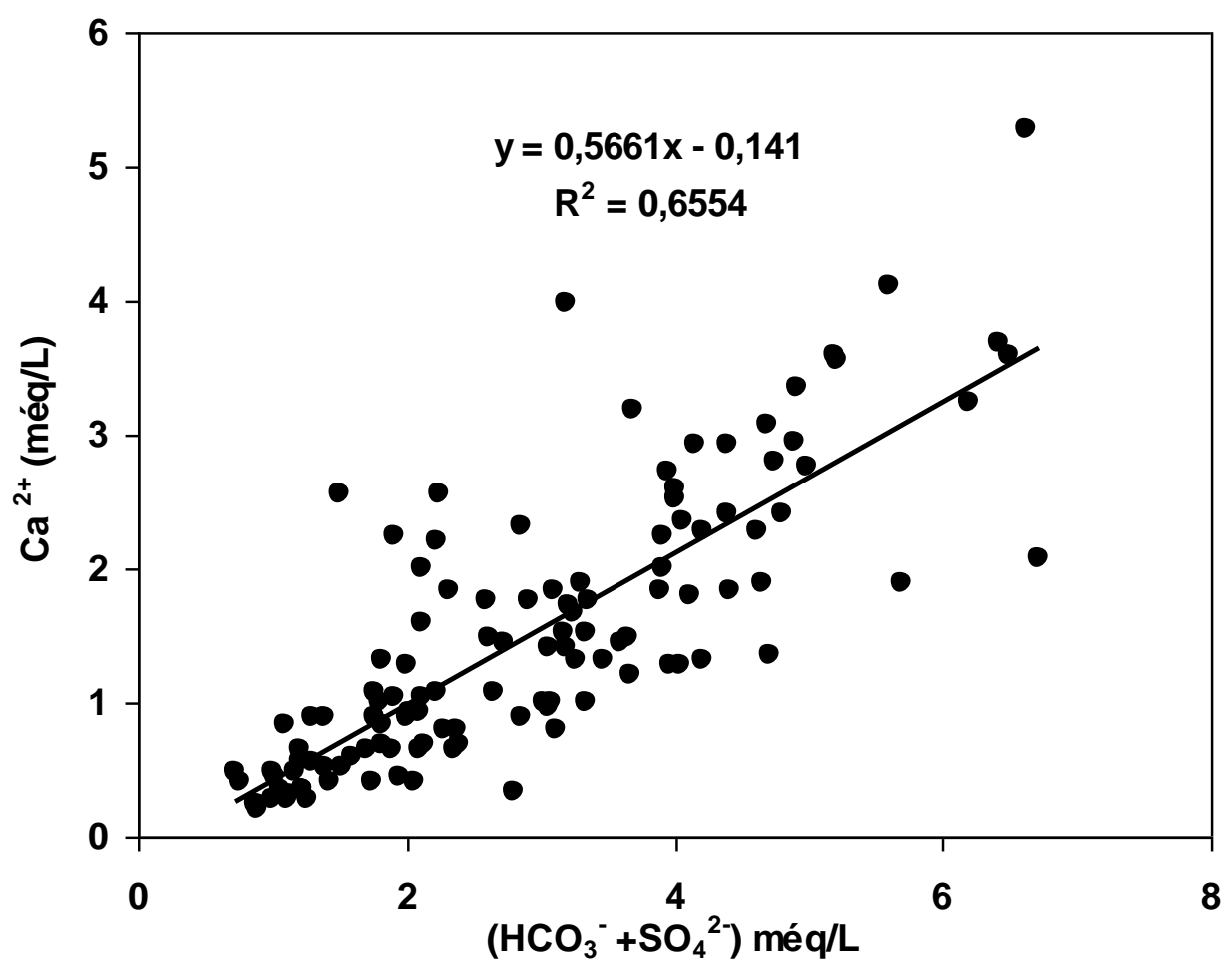

Figure 7: Graphe de corrélation entre $\mathrm{Ca}^{2+}$ et $\left(\mathrm{HCO}_{3}{ }^{-}+\mathrm{SO}_{4}{ }^{2-}\right)$.

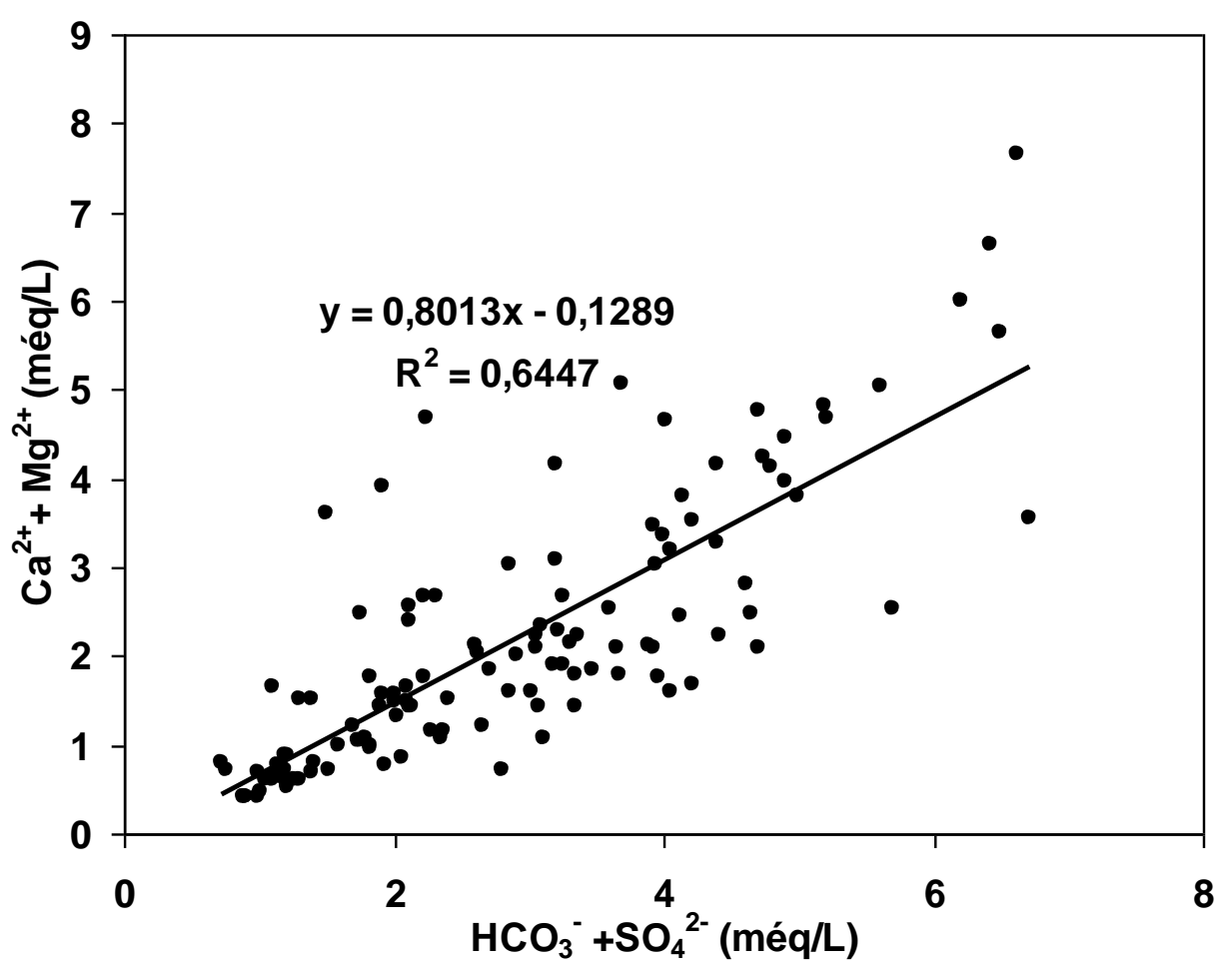

Figure 8 : Diagramme de $\left(\mathrm{Ca}^{2+}+\mathrm{Mg}^{2+}\right)$ en fonction de $\left(\mathrm{HCO}_{3}{ }^{-}+\mathrm{SO}_{4}{ }^{2-}\right)$. 


\section{DISCUSSION}

Le $\mathrm{pH}$ moyen des eaux souterraines est de 6,6. L'intervalle de valeurs de $\mathrm{pH}$ recommandé par l'OMS est 6,5-8,5. Le pH des eaux analysées est presque en-dessous de la limite inférieure de 6,5. Ceci montre le caractère acide de ces eaux. L'acidité des eaux souterraines peut être due à un processus biogéochimique ou anthropogénique. $\mathrm{Ce}$ processus est régi par le $\mathrm{CO}_{2}$ du sol généré à travers la respiration des racines des plantes et de la décomposition de la matière organique (Kortatsi et al., 2008 ; Matini et al., 2009). La présence de forêts galeries et de forêts classées et une pluviométrie relativement abondante, favorisent la décomposition de la matière organique dans la région des Lacs. Les concentrations élevées en fer et en manganèse par rapport aux normes OMS (2006), donnent souvent à l'eau une couleur rougeâtre. Cette couleur rougeâtre est souvent à l'origine du rejet de ces forages par les populations. Or le fer est généralement conseillé aux hommes qui ont une carence en fer (Kouassi et al., 2012). Le rejet de ces points d'eau est souvent d'ordre esthétique et/ou organoleptique. A cet effet, un excès de minéralisation de l'eau de certains forages donne un gout salé à l'eau lors de sa consommation. Ce goût salé de l'eau est également l'une des causes d'abandon des forages par les populations qui en réalité ne sont pas contaminés ni pollués au profit des eaux de marigots et autres rivières dont la qualité est douteuse. Les points d'eau de Subiakro et d'Akpessekro sont les seuls points qui présentent des concentrations en nitrates au-dessus de la norme OMS (50 mg/L). Ces eaux ne doivent pas être utilisées pour la boisson afin de prévenir des risques potentiels de maladies. En effet, l'ingestion d'une eau riche en nitrates peut provoquer des maladies telles que la méthémoglobine chez les enfants. De ce fait, ces deux points d'eau doivent être abandonnés. Par ailleurs, selon Alagbe (2006), la présence de concentrations élevées en nitrates dans les eaux peut indiquer aussi que l'eau contient des bactéries très dangereuses et d'autres polluants. Le nitrate est polluant commun des eaux souterraines, il est généralement issu de l'agriculture (utilisation des engrais), des eaux usées domestiques et des fumures animales (Yuan et al., 2017). La zone d'étude étant une zone agricole, la présence des nitrates dans les eaux serait due aux activités agricoles utilisatrices d'engrais chimiques. Une autre source probable serait aussi, la décomposition de la matière organique végétale (Matini et al., 2009).

Les concentrations en ions sulfates et chlorures sont toutes en dessous des normes des potabilités telles que définies par l'OMS. Ce sont des ions qui peuvent dégrader la qualité des eaux de consommation lorsque leurs concentrations sont supérieures aux normes établies. L'origine des ions chlorures peut-être naturelle dans le cas de l'infiltration d'eaux marines. Cependant tel n'est pas le cas dans cette étude. En effet, la zone d'étude est à plus de $250 \mathrm{~km}$ à vol d'oiseau des cotes maritimes. C'est pourquoi, les ions chlorures peuvent être liés aux rejets humains (Matini et al., 2009) et à la décomposition de la matière organique végétale associé au phénomène de pluviolessivage. L'origine probable des sulfates pourrait être l'oxydation de la pyrite (Kortatsi et al., 2008) suivant la réaction :

$$
\mathrm{FeS}_{2}+3,5 \mathrm{O}_{2}+\mathrm{H}_{2} \mathrm{O}=\mathrm{Fe}^{2+}+2 \mathrm{SO}_{4}{ }^{2-}+2 \mathrm{H}^{+}
$$

Cette réaction libère des ions sulfates $\left(\mathrm{SO}_{4}{ }^{2-}\right)$ et du fer dans les eaux souterraines. Les travaux de Coulibaly et al. (2008) ont trouvé un assemblage de minéraux sulfureux tels que la pyrite-chalcopyrite dans le Birimien au centre de la Côte d'Ivoire et notamment dans la zone qui fait l'objet de cette étude. C'est pourquoi l'hypothèse d'une origine géologique des ions sulfates dans la zone semble plausible.

Durant l'évolution des eaux dans l'aquifère, l'une des conséquences de son long temps de séjour, est l'augmentation progressive de la minéralisation. Ce processus utilise le $\mathrm{CO}_{2}$ issu de la décomposition de la matière organique et s'infiltre grâce eaux de pluies dans le sol et dans les nappes. Cette 
réaction chimique va participer à la dissolution de la matrice rocheuse constituée de minéraux silicatés. L'hydrolyse acide de ces minéraux libère des ions dans les eaux souterraines et en majorité des ions bicarbonates. Ainsi, l'hydrolyse des silicates s'avère être le principal mécanisme qui permet de libérer les ions alcalins au détriment des cations. C'est ce qui explique sans aucun doute la prédominance des bicarbonates dans la charge saline des eaux par rapport aux cations dont la mise en solution provient de la dissolution des carbonates secondaires. En conséquence, la présence des ions bicarbonates, calcium, magnésium et sulfates dans les eaux serait en majorité liée au processus hydrogéochimique qui est l'hydrolyse des minéraux silicatés et la dissolution des carbonates néoformés.

L'ACP et la CAH ont permis de mettre en évidence trois principaux mécanismes hydrogéochimiques responsables de l'évolution de la minéralisation des eaux dans les aquifères de fissures de la zone d'étude. Il s'agit dans un premier temps de la destruction de la végétation et de la décomposition de la litière qui produisent aussi bien des éléments tels que les nitrates et les chlorures mais aussi des cations $\left(\mathrm{Ca}^{2+}, \mathrm{Mg}^{2+}, \ldots\right)$. De même, l'usage des intrants agricoles (fertilisants) et la décomposition de la matière organique due à la déforestation pourraient produire une grande quantité de nitrates qui serait par la suite entraînée vers les nappes par le pluviolessivage et enrichir ces eaux en nitrates. Des études récentes ont également montré que les activités anthropiques telle que l'utilisation des engrais chimiques, les dépôts d'ordures ménagères et les effluents domestiques ont une influence sur la composition chimique des eaux souterraines (Ahoussi et al., 2008 ; Soro et al., 2013 ; Yuan et al., 2017). Ce processus est contrôlé par l'infiltration directe des éléments dans la nappe occasionnant une pollution azotée des eaux souterraines. Le deuxième mécanisme hydrogéochimique à la base de la minéralisation en éléments métalliques des eaux est l'oxydo-réduction. La présence dans les eaux souterraines de concentrations élevées de fer et de manganèse est liée à ce mécanisme hydrogéochimique non pas à une origine anthropogénique (Tay et Kortatsi, 2008). Par ailleurs, la source de Fer dans les eaux souterraines pourrait être due à l'altération de la biotite (K $(\mathrm{Fe}, \mathrm{Mg})_{3}(\mathrm{OH})_{2} \mathrm{AlSi}_{3} \mathrm{O}_{10}$ (Sakyi et al., 2016) et à l'oxydation de la pyrite (Kortatsi et al., 2008). Il en est de même pour le Mn qui est susceptible d'être issu du processus naturel d'interaction eau-roche (Boateng et al., 2015 ; Sakyi et al., 2016 ; Tay et al., 2017). Enfin, la minéralisation naturelle par altération du réservoir dans le temps et précipitation des ions issus de la dissolution des silicates reste le processus majeur qui gouverne le chimisme des eaux souterraines dans la région des Lacs. Ce mécanisme est catalysé par la présence dans l'eau du $\mathrm{CO}_{2}$ provenant de la décomposition de la matière organique et qui s'infiltre dans les nappes à la faveur des précipitations. $\mathrm{Ce} \mathrm{CO}_{2}$ facilite la réaction d'hydrolyse des minéraux silicatés et permet la libération des ions $\mathrm{HCO}_{3}{ }^{-}$ainsi que certains cations. C'est pour cette raison que l'eau des nappes de fissures, bien que relativement peu minéralisée, présente de fortes teneurs en $\mathrm{HCO}_{3}^{-}$. Cette abondance des ions bicarbonates dans les eaux souterraines pourrait s'expliquer selon Kouassi et al., (2012) par l'hydrolyse des feldspaths qui sont des constituants des roches aquifères. Les cations dominants qui s'associent à ces ions bicarbonates sont le $\mathrm{Ca}^{2+}$ et le $\mathrm{Mg}^{2+}$ (Kouassi et al., 2012). La dissolution du feldspath potassique et des micas produit des ions $\mathrm{K}^{+}$, $\mathrm{Mg}^{2+}$ et $\mathrm{HCO}_{3}{ }^{-}$dans les eaux souterraines. Ces processus chimiques se traduisent par les réactions chimiques suivantes (Yuan et al., 2017) :

$$
\begin{aligned}
& 4 \mathrm{KAlSi}_{3} \mathrm{O}_{8} \quad(\mathrm{~K} \text {-feldspaths })+4 \mathrm{CO}_{2} \\
& +22 \mathrm{H}_{2} \mathrm{O} \rightarrow \mathrm{Al}_{4}\left(\mathrm{Si}_{4} \mathrm{O}_{10}\right)(\mathrm{OH})_{8}+8 \mathrm{H}_{4} \mathrm{SiO}_{4}+ \\
& 4 \mathrm{~K}^{+}+4 \mathrm{HCO}_{3}^{-} \quad 4 \mathrm{KMg}^{+} \mathrm{AlSi}_{3} \mathrm{O}_{10} \quad(\mathrm{OH})_{2} \\
& (\mathrm{micas})+28 \mathrm{CO}_{2}+30 \mathrm{H}_{2} \mathrm{O} \rightarrow \mathrm{Al}_{4}\left(\mathrm{Si}_{4} \mathrm{O}_{10}\right)(\mathrm{OH})_{8} \\
& +8 \mathrm{H}_{4} \mathrm{SiO}_{4}+4 \mathrm{~K}^{+}+28 \mathrm{HCO}_{3}^{-}+12 \mathrm{Mg}^{2+}
\end{aligned}
$$


$\mathrm{Au}$ total, trois processus hydrogéochimiques sont à la base du mécanisme de la minéralisation des eaux souterraines de la zone d'étude. Le processus majeur de la minéralisation des eaux est l'hydrolyse des minéraux silicates qui fournit la grande quantité d'ions. L'oxydo-réduction est à la base de la précipitation du fer et du manganèse dans les eaux. Enfin, les activités anthropiques telles que l'usage d'engrais chimiques dans l'agriculture, les eaux usées et les dépôts d'ordures sont à la base de la présence des nitrates et des chlorures dans les eaux souterraines.

\section{Conclusion}

La présente étude avait pour objectif de comprendre les mécanismes qui contrôlent l'hydrochimie des eaux souterraines de la région des Lacs (centre de la Côte d'Ivoire) à partir des techniques d'analyse statistique multivariée (ACP et $\mathrm{CAH}$ ). Au niveau des paramètres physiques mesurés in situ, la température moyenne des eaux des forages est de $27,21^{\circ} \mathrm{C}$. Les eaux souterraines sont acides avec un $\mathrm{pH}$ moyen de 6,6. La conductivité électrique moyenne est de $331 \mu \mathrm{S} / \mathrm{cm}$ avec des valeurs extrêmes comprises entre 11,2 et 1097 $\mu \mathrm{S} / \mathrm{cm}$. Dans l'ensemble, les eaux sont moyennement minéralisées et aptes à la consommation. Cependant certains points d'eau présentent des valeurs critiques des nitrates et du fer. Deux points d'eau ont des concentrations nitrates supérieures à la norme OMS. L'eau de ces ouvrages ne doit pas être consommée à cause des risques sanitaires qu'elle présente. Par ailleurs, la couleur rougeâtre due à l'excès de fer et de manganèse dans certains points d'eau peut entrainer l'abandon de ces derniers par les populations. L'ACP a montré que les trois premiers facteurs totalisent $75 \%$ de la variance totale exprimée par les 13 variables. Par ailleurs, l'analyse combinée de l'ACP et la CAH suggère que les caractéristiques hydrogéochimiques des eaux souterraines sont principalement contrôlées par trois principaux processus dont le principal est l'hydrolyse acide des minéraux silicatés. De manière spécifique, les minéraux tels que les feldspaths potassiques, la biotite, les micas produisent l'essentiel des éléments chimiques des eaux souterraines tels que le $\mathrm{Ca}^{2+}, \mathrm{Mg}^{2+}$, $\mathrm{SO}_{4}{ }^{3-} \mathrm{HCO}_{3}{ }^{-}$. Les concentrations de nitrates et de chlorures dans les eaux sont attribuées à l'impact des activités anthropiques telles que l'usage intensif des engrais chimiques, les eaux usées et le cycle biogéochimique (destruction de la végétation, décomposition de la matière organique). Par ailleurs, les concentrations élevées de fer et de manganèse dans les eaux est lié au phénomène d'oxydoréduction. Cette étude a permis de mettre en lumière les processus qui régissent l'hydrochimie des eaux souterraines. Ces résultats pourront aider les autorités en charge l'approvisionnement en eau des populations pour une meilleure planification et utilisation rationnelle des ressources en eaux souterraines.

\section{CONFLIT D'INTERETS}

Les auteurs déclarent qu'il n'y a aucun conflit d'intérêts.

\section{CONTRIBUTIONS DES AUTEURS}

GS a fait la collecte, le traitement statistique et la rédaction du manuscrit; TDS a fait le montage et la rédaction du manuscrit ; NMRF, OAA ont participé à la rédaction et la relecture du manuscrit; NS a coordonné l'étude, la lecture et la correction du manuscrit.

\section{REMERCIEMENTS}

Nos remerciements vont à l'endroit des responsables de la Direction territoriale de l'hydraulique Yamoussoukro, pour la mise à notre disposition des données et des moyens d'accès sur les ouvrages hydrauliques.

\section{REFERENCES}

Ahoussi KE, Soro N, Soro G, Lasm T, Oga MS, Zadé SP. 2008. Groundwater pollution in Africans biggest towns: case of the town of Abidjan (Côte d'Ivoire). 
European Journal of Scientific Research, 20(2): 302-316. http://www.eurojournals.com/ejsr.htm

Akoteyon IS. 2013. Characterization of groundwater hydrochemistry and quality assessment in Eti-Osa, Lagos-Nigeria. Ethiopian Journal of Environmental Studies and Management, 6(2): 201-214. DOI:

http://dx.doi.org/10.4314/ejesm.v6i2.11

Alagbe SA. 2006. Preliminary evaluation of hydrochemistry of the Kalambaina Formation, Sokoto Basin, Nigeria. Environ. Geol., 51: 39-45. DOI: https://doi.org/10.1007/s00254-0060302-5

Alaya MB, Zemni T, Mamou A, Zargouni F. 2014. Acquisition de salinité et qualité des eaux d'une nappe profonde en Tunisie : approche statistique et géochimique. Hydrological Sciences Journal, 59(2): 395-419. DOI : https://doi.org/10.1080/02626667.2013.8 70663

Amadou H, Laouali MS, Manzola AS. 2014. Caractérisation hydrochimique des eaux souterraines de la région de Tahoua (Niger). Journal of Applied Biosciences, 80: $\quad 7173-7185 . \quad$ DOI : http://dx.doi.org/10.4314/jab.v81i1.6

Sakyi PA, Asare R, Fynn OF, Osiakwan GM. 2016. Assessment of Groundwater Quality and its Suitability for Domestic and Agricultural Purposes in parts of the Central Region, Ghana. West African Journal of Applied Ecology, 24(2):6789. http://apps.ug.edu.gh/wajae.

Boateng T K, Opoku F, Acquaah OS, Akoto O. 2015. Pollution evaluation, sources and risk assessment of heavy metals in hand-dug wells from Ejisu-Juaben Municipality, Ghana. Environ Syst Res, 4:18.

DOI: https://doi.org/10.1186/s40068-0150045-y

Calow CR, MacDoanald AM, Nicol AL, Robins NS. 2010 .Ground water security and drought in Africa: Linking availability, access and demand. Ground Water, $\quad 48(2): 246-256 . \quad$ DOI: http://doi.org/10.1111/j.17456584.2009.00558.x

Coulibaly Y, Boiron MC, Cathelineau M, Kouamelan AN. 2008. Fluid immiscibility and gold deposition in the birimian quartz veins of the Angovia deposit (Yaouré, Ivory Coast). Journal of African Earth Sciences, 50(2-4):234254.

DOI: http://doi.org/10.1016/j.jafrearsci.2007.0 9.014

Cloutier V, Lefebvre R, Therrien R, Savard MM. 2008. Multivariate statistical analysis of geochemical data as indicative of the hydrogeochemeical evolution of groundwater in a sedimentary rock aquifer system. Journal of Hydrology, 353: 294-313. DOI:

http://doi.org/10.1016/j.jhydrol.2008.02. 015

Engalenc M. 1978. Méthodes d'étude et de recherche de l'eau souterraine des roches cristallines de l'Afrique de l'Ouest. Comité interafricain d'études hydrauliques (CIEH), série hydrogéologie, GeohydrauliqueLaboratoire Central d'Hydraulique de France.

Garry B. 2007. Étude des processus d'écoulements de la zone non saturée pour la modélisation des aquifères karstiques : Expérimentation hydrodynamique et hydrochimique sur les sites du Laboratoire Souterrain à Bas Bruit (LSBB) de Rustrel et de Fontaine de Vaucluse. Thèse de Doctorat, Université d'Avignon et des pays de Vaucluse, France, p 206.

Güler C, Thyne GD, McGray JE, Turner AK. 2002. Evaluation of graphical and multivariatestatistical methods for classification of water chemistry data. Hydrogeology Journal, 10(4): 455-474. https://doi.org/10.1007/s10040-0020196-6. 
Hussein MT. 2004. Hydrochemical evaluation of groundwater in the Blue Nile Basin, eastern Sudan, using conventional and multivariate techniques. Hydrogeology Journal, 12: 144-158. DOI: https://doi.org/10.1007/s10040-0030265-5

Institut National de la Statistique . 2014. Recensement général de la population et de l'Habitat (RGPH) 2014. Données socio-démographiques et économiques: Région des lacs.

Jiang Y, Wu Y, Groves C, Yuan D, Kambesis P. 2009. Natural and anthropogenic factors affecting groundwater quality in the Nandong karst underground river system in Yunan, China. Journal of Contaminant Hydrology, 109: 49-61. DOI:

https://doi.org/10.1016/j.jconthyd.2009.0 8.001

Kim KH, Yun ST, Choi BY, Chae GT, Joo Y, Kim K., Kim HS. 2009. Hydrochemical and multivariate statistical interpretations of the spatial controls of nitrate concentrations in a shallow alluvial aquifer around oxbow lakes (Osong area, central Korea). Journal of Contaminant Hydrology, 107:114-127. DOI: https://doi.org/10.1016/j.jconthyd.2009.0 4.007

Kouassi MA, Ahoussi KE, Koffi YB, Ake YA, Biemi J. 2012. Caractérisation hydrogéochimique des eaux des aquifères fissurés de la zone GuigloDuekoué (Ouest de la Côte d'Ivoire). Int. J. Biol. Chem. Sci., 6(1): 504-518. DOI: http://dx.doi.org/10.4314/ijbcs.v6i1.45

Kortatsi BK, Tay CK; Anornu G., Hayford E., Dartey GA. 2008. Hydrogeochemical evaluation of groundwater in the lower Offin basin, Ghana. Environ. Geol., 53(8):1651-1662.

DOI : https://doi.org/10.1007/s00254-0070772-0

Leblond P. 1984. Contribution aux études hydrogéologiques en Côte d'Ivoire. Région de Yamoussoukro (Station expérimentale de l'ENSTP). Thèse de Doctorat $3^{\grave{e}}$ cycle, Université de Bordeaux 1, France, p.150.

Matini L, Moutou1 JM, Kongo-Mantono MS. 2009. Evaluation hydro-chimique des eaux souterraines en milieu urbain au Sud-Ouest de Brazzaville, Congo. Afrique SCIENCE, $\quad \mathbf{0 5}(1): 82-98$. https://www.ajol.info/index.php/afsci/art icle/view/61709.

Monjerezi M, Vogt RD, Aagaard P, Saka JDK. 2012. The hydro-geochemistry of groundwater resources in an area with prevailing saline groundwater, lower Shire Valley, Malawi. Journal of African Earth Sciences, 68: 67-81. DOI: https://dx.doi.org/10.1016/j.jafrearsci.20 12.03.012

N'guessan A. 1985. Contribution à l'hydrogéologie de la région Centrale de la Côte d'Ivoire et analyse statistique des résultats de forage. Thèse de Doctorat 3è cycle, Université de Franche-Comté, Besançon, France, p.154.

OMS. 2006. Normes de l'OMS sur l'eau potable. OMS.

Sojka M, Siepak M, Ziola A, Frankowski M, Murat-Blazejewska S, Siepak J. 2008. Application of multivariate statistical techniques to evaluation of water quality in the Mala Welna river (Western Poland). Environ Monit Assess, 147:159170.

DOI:

https://doi.org/10.1007/s10661-0070107-3

Soro G. 2010. Evaluation quantitative et qualitative des ressources en eaux souterraines de la région des Lacs (centre de la Côte d'Ivoire) : Hydrogéologie et hydrochimie des aquifères continus du district de Yamoussoukro et du département de Tiébissou. Thèse unique de Doctorat, Université de Cocody, Côte d'Ivoire, p. 254.

Soro G, Soro N, Ahoussi KE, Lasm T, Kouamé FK, Soro TD, Biémi J. 2010. 
Evaluation des propriétés hydrauliques des aquifères fracturés des formations cristalline et métamorphique dans la région des Lacs (centre de la Côte d'Ivoire). Estudios Geológicos, $\mathbf{6 6}(2)$ : 227-242.

DOI: https://doi.org/10.3989/egeol.40123.093

Soro TD, Ahoussi KE, Oga YMS, Soro G, Soro N. 2013. A multivariate statistical analysis of groundwater chemistry data in the Highest Bandama basin at Tortiya (Northern Côte d'Ivoire). Earth Resources, 1(3): 72-77. DOI: https://doi.org/10.12966/er.09.03.2013

Soule de Lafont D. 1956. Le précambrien moyen et supérieur de Bondoukou (Côte d'Ivoire). Bulletin de la Direction Fédérale des Mines et de la Géologie, 18: 1-174.

Tay CK, Hayford EK, Hodgson IOA. 2017. Application of multivariate statistical technique for hydrogeochemical assessment of groundwater within the Lower Pra Basin, Ghana. Appl Water Sci., 7(3): 1131-1150. DOI: https://doi.org/10.1007/s13201-017-5406

Tay CK, Kortatsi B. 2008. Groundwater Quality Studies: A Case Study of the Densu Basin, Ghana. West African
Journal of Applied Ecology, 12. DOI: https://doi.org/10.4314/wajae.v12i1.4576 0

Yacé I. 2002. Initiation à la géologie. L'exemple de la Côte d'Ivoire et de l'Afrique de l'Ouest. Pétrologie, Géologie régionale. Edition. CEDA, SODEMI.

Yidana SM, Banoeng-Yakubo B, Akabzaa TM. 2010. Analysis of groundwater quality using multivariate and spatial analyses in the Keta basin, Ghana. Journal of African Earth Sciences, 58: 220-234.

DOI: https://doi.org/10.1016/j.jafrearsci.2010. 03.003

Yidana SM, Yiran GB, Sakyi PA, Nude PM, Banoeng-Yakubo B. 2011. Groundwater evolution in the Voltaian Basin, Ghana; An application of multivariate statistical analyses to hydrochemical data. Natural Science, 3(10): 837-854. DOI: https://doi.org/10.4236/ns.2011.310109

Yuan J, Xu F, Deng G, Tang Y, Li P. 2017. Hydrogeochemistry of shallow groundwater in a karst aquifer system of Bijie City, Guizhou province. Water, 9(625): $1-16 . \quad$ DOI: https://doi.org/10.3390/w9080625. 